\title{
3D surfactant-dispersed graphenes as cathode interfacial materials for organic solar cells
}

\author{
Fei Pan ${ }^{1,2,4}$, Song $\mathrm{Bai}^{1}$, Xian $\mathrm{Wei}^{1}$, Yingfen $\mathrm{Li}^{1}$, Dianyong Tang ${ }^{3}$, Xiwen $\mathrm{Chen}^{5}$, Menglan $\mathrm{Lv}^{1,2^{*}}$ and \\ Yongfang $\mathrm{Li}^{2,4^{*}}$
}

\begin{abstract}
Graphene dispersions in low-boiling-point green solvents have wide applications in coatings, conducting inks, batteries, electronics and solar cells. Two three-dimensional (3D) cathode interfacial materials (CIMs) $(1,3,5,7,9,11,13,15$-octa-(9-bis(30-( $N, N$-dimethylamino)propyl)-2,7-fluorene)-vinylpentacyclo-octasiloxane) (POSSFN) and (1,3,5,7-tetra-(9-bis(30-(N,N-dimethylamino)propyl)-2,7fluorene)-adamantane) (ADMAFN) are excellent surfactants for dispersing graphene in ethanol at the concentration of 0.97-1.18 $\mathrm{mg} \mathrm{mL}^{-1}$, in agreement with their calculated large adsorption energies on graphene. The results of electron spin resonance, Raman, scanning Kelvin probe microscopy and $\mathrm{X}$ ray photoelectron spectroscopy measurements indicate that the amino groups could $n$-dope graphene or form dipole interaction with graphene. The two 3D-surfactant-based graphene composites (POSSFN-G and ADMAFN-G) can work as high-performance CIMs in organic solar cells (OSCs), which improve the power conversion efficiency (PCE) of the OSCs based on PM6:Y6 to 15.9\%-16.1\%. ADMAFN forms dipole interaction with graphene in ADMAFN-G and the composite CIM delivers high PCE of $\mathbf{1 6 . 1 1 \%}$ in the OSCs, while POSSFN forms n-doped composition with graphene in POSSFN-G which works well as thicker CIM film in the OSCs.
\end{abstract}

Keywords: organic solar cells, interfaces, 3D cathode interfacial materials, graphene composites

\section{INTRODUCTION}

Organic solar cells (OSCs) have underwent significant progress recently and become one of the promisingly renewable energy technologies [1,2]. Organic-semiconductor-based photovoltaic materials offer a flexible mechanical property, versatile chemical structure, light weight and low cost. These characteristics make OSCs potential energy source in transparent power-generating glass, zero-emission buildings, new-energy vehicles, and energy cycle in public places.

Two main types of organic semiconductors are used in the OSCs, which are n-type small molecule organic acceptor and p-type conjugated polymer donor [3-9]. The complementary absorption and electronic energy level matching of the donor and acceptor materials are of crucial importance for achieving high power conversion efficiency (PCE) of the OSCs [8-9]. In addition, the electrode interfacial materials are also very important for high-performance OSCs [10-16]. Especially, the alcohol/ water soluble cathode interfacial materials (CIMs), which can lower the work function of cathode, suppress the charge recombination and improve the charge extraction, have attracted great attention in the development of OSCs [10-16].

Graphene possesses high charge carrier mobility, mechanical flexibility, compatibility with solution processing and tunable Fermi energy [17-20]. Very recently, we developed a new CIM PDINO-G by dispersing graphene in a typical water/alcohol soluble CIM PDINO $((N, N-$ dimethyl-ammonium $\mathrm{N}$-oxide) propyl perylene diimide) [21]. The graphene was $n$-doped by PDINO in PDINO-G which became an excellent CIM for OSCs. To further explore the application of graphene composites as CIMs

\footnotetext{
${ }^{1}$ Guizhou Institute of Technology, Guiyang 550003, China

${ }^{2}$ Beijing National Laboratory for Molecular Sciences, CAS Key Laboratory of Organic Solids, Institute of Chemistry, Chinese Academy of Sciences, Beijing 100190, China

${ }^{3}$ Chongqing Engineering Laboratory of Targeted and Innovative Therapeutics, International Academy of Targeted Therapeutics and Innovation, Chongqing University of Arts and Sciences, Chongqing 402160, China

${ }^{4}$ School of Chemical Science, University of Chinese Academy of Sciences, Beijing 100049, China

${ }^{5}$ Guangzhou Polyforte Chemical Technology Co. LTD, Guangzhou 510520, China

* Corresponding authors (emails: lvmenglan@git.edu.cn (Lv M); liyf@iccas.ac.cn (Li Y))
} 
for the OSCs with high performance, it is very important to understand how the structure of traditional water/alcohol soluble CIMs affects the graphene dispersion and the modification of graphene structure.

Previously, we reported a series of three-dimensional (3D) alcohol/water soluble materials with pendant ammonium salts or neutral amines as CIMs, which work similarly to or better than their linear structured CIMs for OSCs $[13,22,23]$. The 3D materials have more pendant functional groups and may possess advantages in comparison with the linear CIMs, such as excellent solubility, prominent processability, and highly spatial molecular structure, which will be beneficial for dispersing the $2 \mathrm{D}$ graphene. With these considerations, in this study, we synthesized two 3D CIMs, POSSFN [23] $(1,3,5,7,9,11,13$, 15-octa-(9-bis(30-(N,N-dimethylamino)propyl)-2,7fluorene)-vinylpentacyclo-octasiloxane) and ADMAFN (1,3,5,7-tetra-(9-bis(30-(N,N-dimethylamino)propyl)-2,7fluorene)-adamantane), as dispersants for graphene, both of which possess the same alcohol-soluble amine groups. They are used for efficiently dispersing graphene to form 3D composite CIMs. The pendant chain self-assembly property of the 3D CIMs affects their interaction with graphene in their composites and impacts on the photovoltaic performance of the OSCs based on the composite CIMs.

\section{EXPERIMENTAL SECTION}

Graphene oxide (GO, thickness: $0.335-1.0 \mathrm{~nm}$; length: 0.5-5.0 $\mu \mathrm{m}$; purity: $>99.9 \%$; layer number: 1$)$, commercial single-layer graphene (micromechanical exfoliation, percentage of single layer: $>95 \%$; thickness: $1-2 \mathrm{~nm}$; length: 5-10 $\mu \mathrm{m}$; layer number: 1-2; purity: $>98 \%)$ and commercial single-layer graphene on silica substrates (CVD) were obtained from Suzhou Tanfeng Graphene Technology Co. LTD. PM6 and Y6 were purchased from Solarmer Materials Inc. and used without further purification. Other chemicals and solvents were obtained from Alfa Aesar, J\&K, and TCI Chemical. Co., respectively. Poly(3,4-ethylenedioxythiophene):polystyrene sulfonate (PEDOT:PSS, PVP Al 4083) was purchased from H. C. Starck. The indium tin oxide (ITO) glass (15 $\Omega$ per square, transmittance $\geq 86 \%$, Nippon sheet glass) was purchased from South China Science \& Technology Company, Ltd. Poly ( $p$-(N,N-dimethylamino) styrene) (PSN) (GPC: $M_{\mathrm{n}}=14.5 \mathrm{kDa} ; M_{\mathrm{w}} / M_{\mathrm{n}}=1.65$ Anal.) was synthesized according to a reference [13]. The $3 \mathrm{D}$ materials ADMAFN and POSSFN were synthesized as described in the Supplementary information (SI) with synthetic routes shown in Schemes S1 and S2. A mixture solution of the surfactant and single-layer graphene power (1-50 wt.\%) in ethanol was sonicated for $60 \mathrm{~min}$ in an ice-water bath (the temperature was controlled at $\left.0-5^{\circ} \mathrm{C}\right)$.

The conventional device structure of the OSCs was ITO/GO/PEDOT:PSS/PM6:Y6/CIMs/Al. Pre-cleaned ITO substrates were treated by UV-ozone treatment for $30 \mathrm{~min}$. GO solution $\left(0.4 \mathrm{mg} \mathrm{mL}^{-1}\right.$ in water) was coated onto the substrates at $5000 \mathrm{rpm}$, and then $10 \mathrm{~nm}$ PEDOT: PSS was coated on the GO film and dried at $150^{\circ} \mathrm{C}$ for $30 \mathrm{~min}$ in air. Next the GO/PEDOT:PSS substrates were transferred into a nitrogen atmosphere glove box. PM6: Y6 (1:1.2 weight ratio in chloroform:chloronaphthalene (99.5:0.5 in volume) solution) blend active layers were spin-coated and annealed at $110^{\circ} \mathrm{C}$ for $5 \mathrm{~min}$. The thickness of the active layer was approximately $110 \mathrm{~nm}$. Afterward the CIMs layers were deposited on the active layer by spin-coating their ethanol solutions at a spincoating rate of $3000 \mathrm{rpm}$. Finally, in a vacuum chamber at a pressure of about $5.0 \times 10^{-5} \mathrm{~Pa}, 100 \mathrm{~nm}$ of aluminum was thermally deposited atop the interlayer. A shadow mask with an effective device area of $0.046 \mathrm{~cm}^{2}$ was used for the completion of the devices.

The current density-voltage $(J-V)$ characteristics were measured by using a computer-controlled Keithley 2400 source measurement unit under 1 sun, AM 1.5G spectrum from a classic solar simulator (Newport 94023A) with a $450 \mathrm{~W}$ Xenon lamp in a glove box filled with nitrogen. The light intensity was calibrated to be $100 \mathrm{~mW} \mathrm{~cm}^{-2}$ by a Newport Oriel $91150 \mathrm{~V}$ reference cell. The external quantum efficiency (EQE) of the OSCs was measured by a Solar Cell Spectral Response Measurement System QE-R3-011 (Enli Technology Co. Ltd., Taiwan). The light intensity at each wavelength was calibrated with a standard single-crystal Si photovoltaic cell.

Molecular weight measurement of PSN was carried out on an Agilent PL-GPC 220 Gel Permeation Chromatography (GPC) instrument, using 1,2,4-trichlorobenzene as the eluent at $160^{\circ} \mathrm{C}$. The bath ultrasonicator was purchased from Shenzhen Pinhuang Science and Technology Ltd. (Model Number: Dongsen DS-040ST), and the maximum ultrasonic power was $240 \mathrm{~W}$, ultrasonic frequency was $40 \mathrm{kHz}$, and max capacity was $10 \mathrm{~L}$. Scanning Kelvin probe microscopy (SKPM) was implemented on a KP Technology SKP5050. The electron spin resonance (ESR) spectra were recorded on a JEOL JES-FA200 ESR spectrometer $(300 \mathrm{~K}, 9.063 \mathrm{GHz}, \mathrm{X}$-band). Microwave power used was $1 \mathrm{~mW}$, and the magnetic field sweep width ranged from 3310 to $3710 \mathrm{G}$. The modulation frequency was $100 \mathrm{kHz}$, and the modulation amplitude 
was 3.5 G. Atomic force microscopy (AFM) measurements were carried out by using a Bruker-ICON2-SYS in a tapping mode. The X-ray photoelectron spectroscopy (XPS) measurements were performed on the ESCALab220i-XL system. The X-ray diffraction (XRD) patterns of the pristine graphite and the graphene films were collected on an Empyrean Rigaku-D/max $2500 \mathrm{~V}$ X-ray diffractometer with $\mathrm{Cu} \mathrm{Ka}$ radiation. Raman spectra were recorded with an Olympus FV1000-IX81 Raman microscope equipped with a 533-nm Ar ion laser. The transient photocurrents of the devices were measured by applying $500-\mathrm{nm}$ laser pulses with a power of $0.354 \mathrm{MW}$. The laser pulses were generated from an optical parametric amplifier (TOPAS-Prime) pumped by a mode-locked Ti: sapphire oscillator seeded regenerative amplifier with a pulse energy of $1.3 \mathrm{~mJ}$ at $800 \mathrm{~nm}$ and a repetition rate of $1 \mathrm{kHz}$ (Spectra Physics Spitfire Ace). The photocurrent produced a transient voltage signal on a $50-\Omega$ resistor, which was recorded by an oscilloscope (Tektronix TDS 3052C).

\section{RESULTS AND DISCUSSION}

\section{Dispersion of graphene in alcohol/water soluble 3D materials}

The reason for designing different kinds of 3D CIMs for graphene dispersion is that: i) to investigate the relationship between different spatial configurations of materials and the dispersion properties of graphene; ii) to study the charge transfer mechanism of the pendant functional groups of the 3D materials with graphene; iii) to verify the effect of the composite materials based on the 3D CIMs and graphene on the cathode surface property in the OSCs.

The molecular dynamics package Forcite plus with the standard periodical boundary conditions and periodic density functional theory [24-26] were conducted to evaluate the molecular dynamics of our molecular design strategy (Figs S1-S3), and the structure of materials adsorbed on single-layered graphene was simulated. The dispersants include two 3D materials ADMAFN and POSSFN, with a linear material poly $(p$ - $(N, N$-dimethylamino) styrene) (PSN) for comparison. The calculation results of the adsorption energies (Table 1) reveal that the 3D materials POSSFN $(-6.37 \mathrm{eV})$ and ADMAFN $(-4.01 \mathrm{eV})$ have much larger adsorption energies than PSN $(-0.64 \mathrm{eV})$ on the single layer graphene. They are also larger than PDINO $(-3.510 \mathrm{eV})[21]$, and a reported CIM N,N-bis [2-(ethanesulfonic acid sodium)]-1,4,5,8naphthalene diimide (NDI-2) $(-1.074 \mathrm{eV})$ [27]. The large
Table 1 Adsorption energies of different surfactants on the graphene surface estimated by periodic density functional theory calculations

\begin{tabular}{cc}
\hline Surfactants & $\begin{array}{c}\text { Adsorption energies on } \\
\text { single-layer graphene surface }(\mathrm{eV})\end{array}$ \\
\hline PDINO & $-3.510[21]$ \\
PSN & -0.64 \\
ADMAFN & -4.01 \\
POSSFN & -6.37 \\
\hline
\end{tabular}

exothermal energy of the 3D materials results in the unique $3 \mathrm{D}$ space structure, $\pi$-conjugated aromatic system and electronic properties of the amino pendant chains that work independently and synergistically. POSSFN has larger adsorption energy than ADMAFN. The pendant functional groups of POSSFN can be self-assembled on the graphene surface while those in ADMAFN are distributed spatially on the graphene surface (Figs 1 and 2).

To measure the graphene-dispersibility of the CIMs, the bath ultrasonic was used to disperse the commercial graphene powder in ethanol with different CIM surfactants (PSN, AMDAFN and POSSFN). Graphene concentrations with the surfactants were calculated based on the absorption spectra shown in Fig. S4 [27]. The graphene concentration increases first and then decreases with the increase of the dispersant concentration, with a maximum graphene concentration of $1.18,0.97$, and $0.45 \mathrm{mg} \mathrm{mL}^{-1}$ at the optimal concentration of ADMAFN $\left(1.00 \mathrm{mg} \mathrm{mL}^{-1}\right)$, POSSFN $\left(1.00 \mathrm{mg} \mathrm{mL}^{-1}\right)$, PSN $\left(4 \mathrm{mg} \mathrm{mL}^{-1}\right)$, respectively. The ability of dispersing graphene in alcohol at these relative high concentrations is highly desirable for many applications where low-boiling-point green solvents are preferred $[28,29]$.

The Tyndall effect (Figs S5 and S6), XPS (Fig. S7), Raman spectroscopy (Fig. S8), XRD (Fig. S9), AFM (Fig. S10), and transmission electron micrographs (TEM) (Fig. S11) were exploited to characterize the dispersion of graphene. The XPS spectrum of the commercial graphene powder displays a prominent $\mathrm{C}=\mathrm{C}$ peak at $284.5 \mathrm{eV}$ and a very weak signal for the $\mathrm{C}-\mathrm{C}$ band $(285.3 \mathrm{eV})$, which are derived from the $\mathrm{sp}^{2}$-hybridized carbon bonds in the graphene lattice and $\mathrm{sp}^{3}$-hybridized carbon bonds respectively, and verify the low content of defects [26,3032]. The Raman spectra of the graphene dispersed in different surfactants with excitation at $532 \mathrm{~nm}$ show the $\mathrm{G}$ band at about $1582 \mathrm{~cm}^{-1}$ of the graphene treated with different dispersants, which is ascribed to the $\mathrm{sp}^{2}$-hybridized carbon bonds in the graphene lattice. The $2 \mathrm{D}$ band at about $2710 \mathrm{~cm}^{-1}$ and the intensity ratio of $2 \mathrm{D} / \mathrm{G}$ confirm the few-layered graphene formed in the com- 
a $\infty$
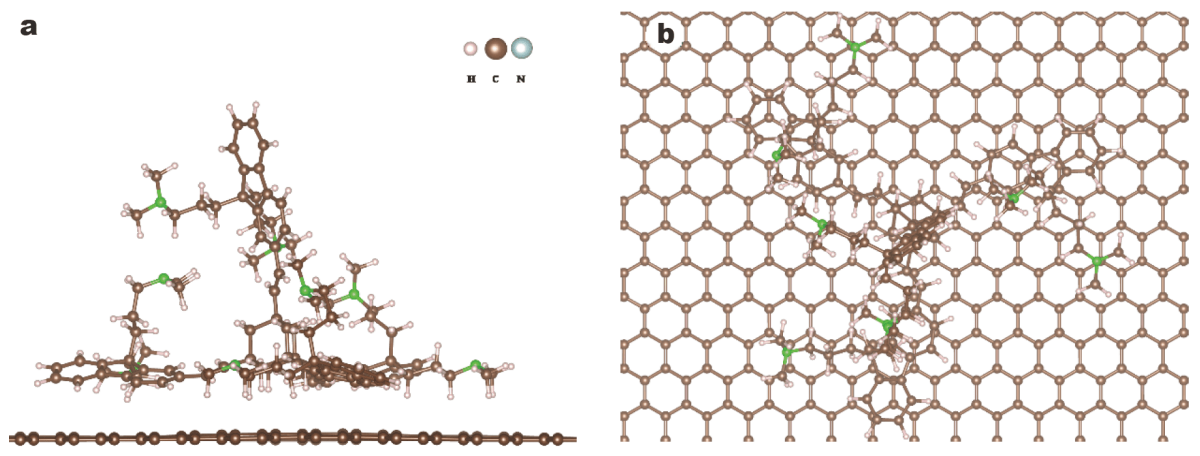

C

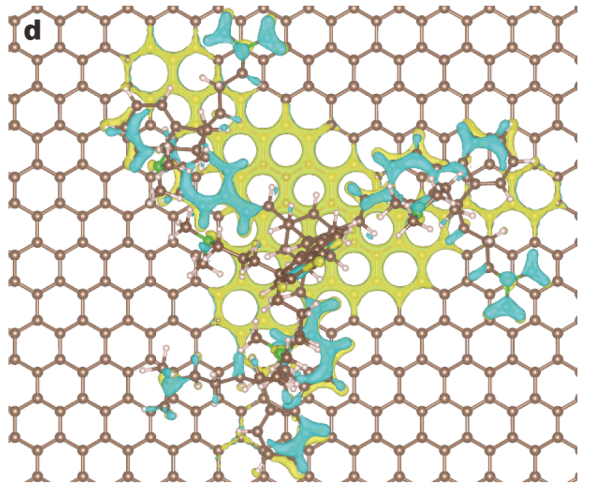

Figure 1 The constructed cluster model of ADMAFN/single layer graphene: (a) side view and (b) top view; (c, d) the calculated charge density differences in the ADMAFN/single layer graphene. The green and yellow regions represent charge accumulation and depletion, respectively, and the isosurface value is 0.04 e $\AA^{-3}$.
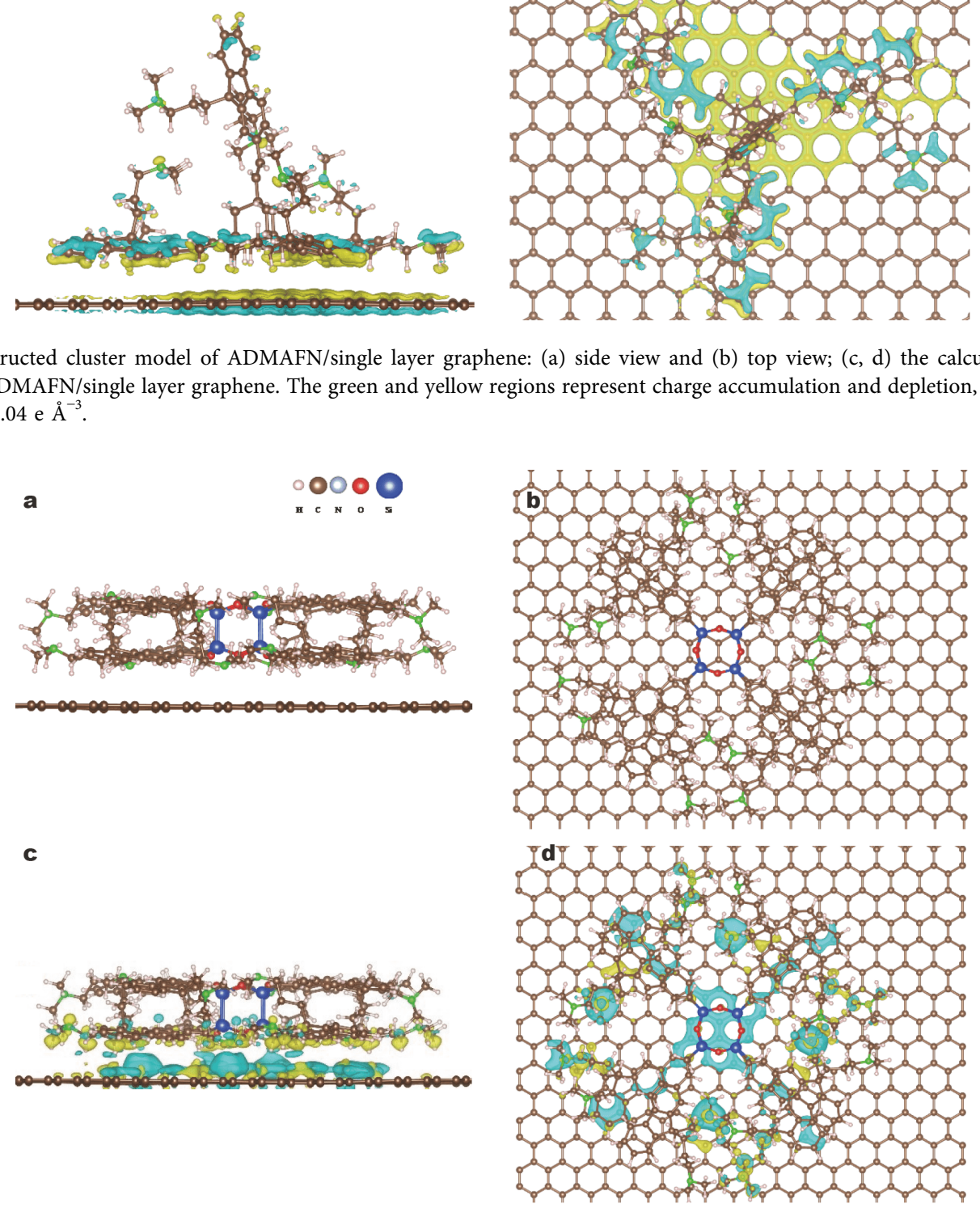

Figure 2 The constructed cluster model of POSSFN/single layer graphene: (a) side view and (b) top view; (c, d) the calculated charge density differences in the POSSFN/single layer graphene. The green and yellow regions represent charge accumulation and depletion, respectively, and the isosurface value is $0.005 \mathrm{e} \AA^{-3}$. 
posites [26,30-32]. The XRD patterns of graphene composites (with $10 \%$ graphene) show broad and weak peak. Note that no graphite peak at $26^{\circ}$ can be seen, which suggests that the dispersions of graphene in PSN, ADMAFN and POSSFN are probably close to the singlesheet level [26,30-32]. The AFM images of the graphene flakes deposited on a mica substrate were measured to characterize the exact number of layers. A lateral size of around $300 \mathrm{~nm}$ with a thickness of about $1.24 \mathrm{~nm}$ was observed. According to the thickness distribution of 50 graphene flakes, the majority of graphene flakes have an average thickness of $1.657 \mathrm{~nm}$, indicating the single-layer feature.

\section{Photovoltaic performance}

The dispersed graphene in PSN, ADMAFN and POSSFN (named as PSN-G, ADMAFN-G and POSSFN-G, respectively) was used to fabricate OSCs as the CIMs (or electron transport layer). The GO-PEDOT:PSS composite was employed as the hole transport layer, as it was reported that the composite showed improved photovoltaic performance than PEDOT:PSS as the anode interlayer [33-35]. We chose copolymer PM6 as the donor and the small molecule n-type organic semiconductor (n-OS) Y6 as the acceptor [8] to construct the OSC devices with a framework of ITO substrates/(GO)-PEDOT:PSS/PM6: $\mathrm{Y} 6 / \mathrm{CIM} / \mathrm{Al}$, and the weight ratio of graphene in the composite CIM was optimized to be about $10 \%$. The molecular structures of PM6, Y6 and the interfacial materials used in this study and architectural structure diagram of the OSCs are displayed in Fig. 3. The $J-V$ curves of the OSCs are shown in Fig. 4a, and the detailed photovoltaic parameters are summarized in Table 2. The PCE of the device increases from $13.82 \%$ (open circuit voltage $\left(V_{\mathrm{oc}}\right)=0.842 \mathrm{~V}$, short-circuit current density $\left(J_{\mathrm{sc}}\right)=$ $23.01 \mathrm{~mA} \mathrm{~cm}^{-2}$, fill factor $(\mathrm{FF})=71.38 \%$ ) for the control device with bare $\mathrm{Al}$ cathode, to $14.77 \%\left(V_{\mathrm{oc}}=0.845 \mathrm{~V}, J_{\mathrm{sc}}\right.$ $=24.46 \mathrm{~mA} \mathrm{~cm}^{-2}, \mathrm{FF}=71.47 \%$ ) for the device with linear material PSN/Al cathode, $15.40 \%\left(V_{\mathrm{oc}}=0.851 \mathrm{~V}, J_{\mathrm{sc}}=\right.$ $24.84 \mathrm{~mA} \mathrm{~cm}^{-2}, \mathrm{FF}=72.87 \%$ ) for the device with $3 \mathrm{D}$ material ADMAFN/Al cathode, and $15.33 \% \quad\left(V_{\text {oc }}\right.$ $\left.=0.853 \mathrm{~V}, J_{\mathrm{sc}}=24.74 \mathrm{~mA} \mathrm{~cm}^{-2}, \mathrm{FF}=72.66 \%\right)$ for the device with 3D material POSSFN/Al cathode. It can be seen that the photovoltaic performances of the 3D CIMs with sphere or regular tetrahedron structure are very similar to that of the linear polymer CIM. By replacing them with the graphene-containing composite CIMs, the resulting device efficiency increases to $15.42 \%\left(V_{\text {oc }}\right.$ $\left.=0.846 \mathrm{~V}, J_{\mathrm{sc}}=24.63 \mathrm{~mA} \mathrm{~cm}{ }^{-2}, \mathrm{FF}=74.01 \%\right)$ for the PSN-G CIM-based OSC, $16.11 \%\left(V_{\mathrm{oc}}=0.845 \mathrm{~V}, J_{\mathrm{sc}}=\right.$ $25.45 \mathrm{~mA} \mathrm{~cm}^{-2}, \mathrm{FF}=74.91 \%$ ) for the ADMAFN-G CIMbased OSC and $15.91 \%\left(V_{\mathrm{oc}}=0.849 \mathrm{~V}, J_{\mathrm{sc}}=25.34\right.$ $\mathrm{mA} \mathrm{cm}^{-2}, \mathrm{FF}=73.93 \%$ ) for the POSSFN-G CIM-based OSC. Fig. $4 \mathrm{~b}$ shows the EQE spectra of the corresponding OSCs. The integral current density values $\left(J_{\text {calc. }}\right)$ from the EQE data agree well with the $J_{\mathrm{sc}}$ values measured from the $J-V$ curves (see Table 2), indicating the reliability of our measurement on the photovoltaic performance.

\section{Characterization of the graphene dispersion}

To further investigate the work mechanism of the graphene-containing $3 \mathrm{D}$ composite CIMs in improving the photovoltaic performance of the OSCs, we measured the conductivity of the CIMs and the work function of various electrodes modified by the CIMs. The conductivities of the graphene-containing composites were measured by

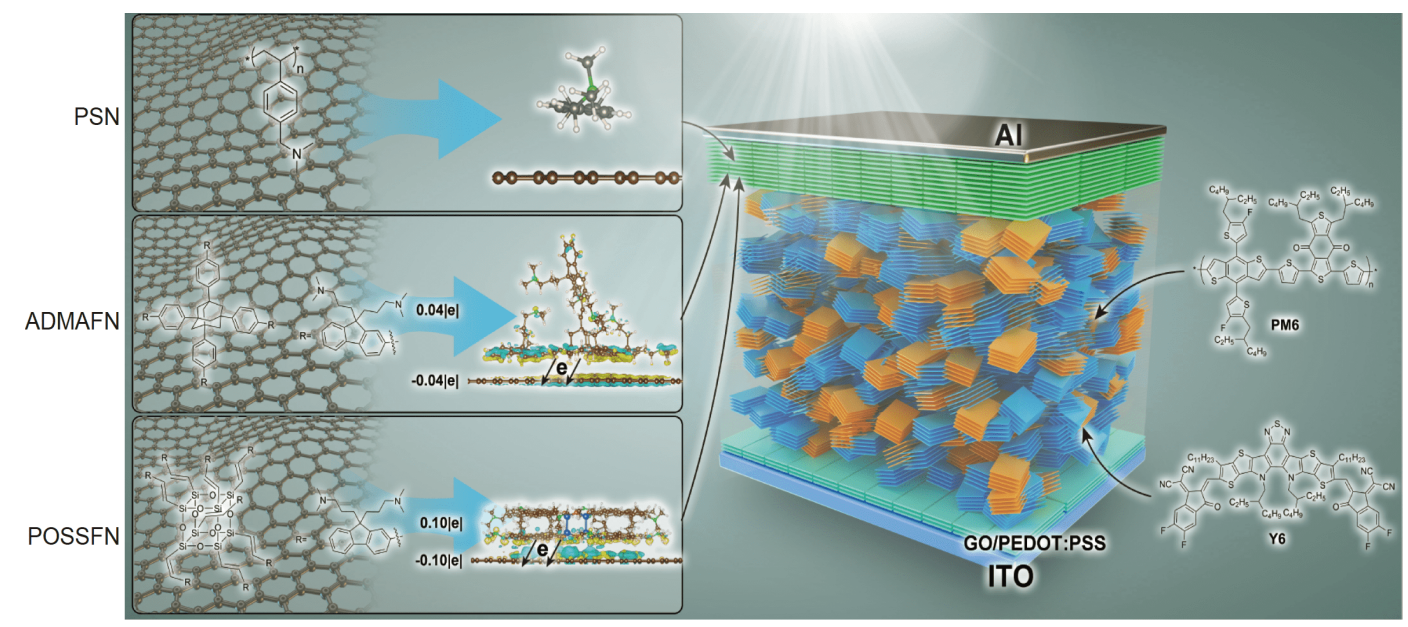

Figure 3 Molecular structures of the photovoltaic and interfacial materials used in this study and the schematic diagram of the OSCs device. 

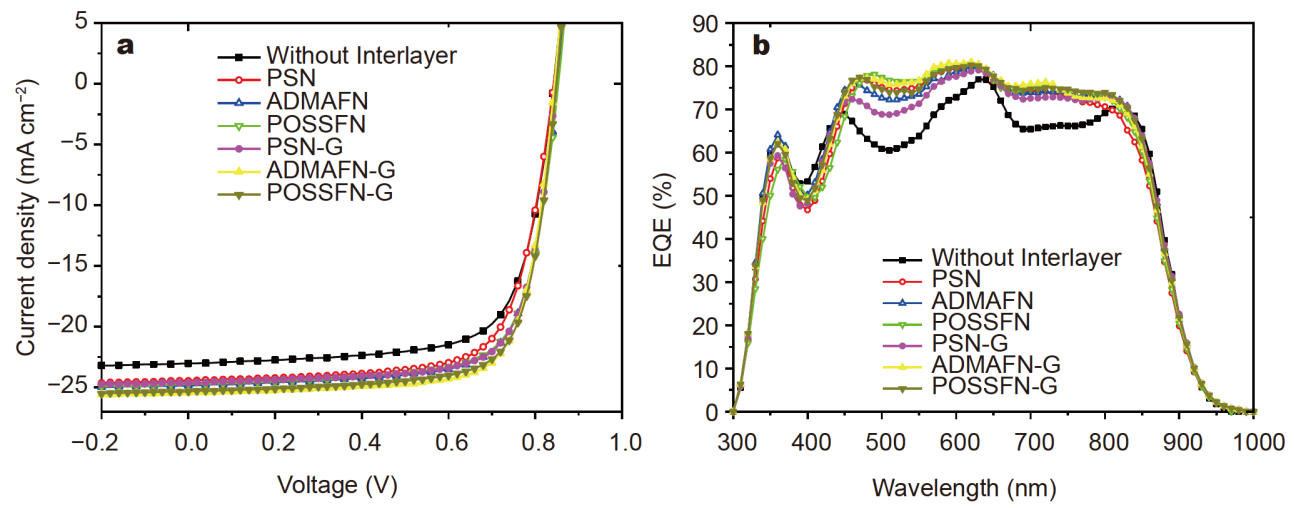

Figure 4 Photovoltaic performances of the OSCs based on PM6:Y6. (a) J-V curves of the OSCs with different CIMs under the illumination of AM1.5G, $100 \mathrm{~mW} \mathrm{~cm}^{-2}$. (b) The external quantum efficiency spectra of the corresponding OSCs.

Table 2 Photovoltaic performance data of the OSCs based on PM6:Y6 with different CIM/Al cathodes under the illumination of AM 1.5 G, $100 \mathrm{~mW} \mathrm{~cm}^{-2}$. The devices structure is ITO/(GO)-PEDOT:PSS/PM6:Y6/CIMs/Al.

\begin{tabular}{|c|c|c|c|c|c|}
\hline CIM/Al cathode & $V_{\mathrm{oc}}(\mathrm{V})$ & $J_{\mathrm{sc}}\left(J_{\mathrm{calc}}{ }^{\mathrm{a}}\right)\left(\mathrm{mA} \mathrm{cm}{ }^{-2}\right)$ & FF (\%) & $\mathrm{PCE}_{\max }(\%)$ & $\operatorname{PCE}_{\text {avg }}(\%)$ \\
\hline $\mathrm{Al}$ & 0.842 & $23.01(22.09)$ & 71.38 & 13.82 & $13.6 \pm 0.2$ \\
\hline $\mathrm{PSN} / \mathrm{Al}$ & 0.845 & $24.46(23.48)$ & 71.47 & 14.77 & $14.5 \pm 0.3$ \\
\hline ADMAFN/Al & 0.851 & $24.84(23.85)$ & 72.87 & 15.40 & $15.2 \pm 0.2$ \\
\hline POSSFN/Al & 0.853 & $24.74(23.75)$ & 72.66 & 15.33 & $15.1 \pm 0.3$ \\
\hline PSN-G/Al & 0.846 & $24.63(23.40)$ & 74.01 & 15.42 & $15.2 \pm 0.2$ \\
\hline ADMAFN-G/Al & 0.845 & 25.45 (24.09) & 74.91 & 16.11 & $15.9 \pm 0.2$ \\
\hline POSSFN-G/Al & 0.849 & $25.34(24.01)$ & 73.93 & 15.91 & $15.7 \pm 0.2$ \\
\hline
\end{tabular}

a) The $J_{\text {calc }}$ is from the external quantum efficiency spectrum.

the Space Charge Limited Current (SCLC) method [36], and the device structures were ITO/graphene-containing composites/Al. In the region of Ohmic contact where the slope of the log-log plot is 1 , the conductivity data is extracted (Fig. S12). The results illustrate that with the addition of graphene, the insulating materials (PSN, ADMAFN and POSSFN) become conductive, and the conductivities increase to $3.05 \times 10^{-5} \mathrm{~S} \mathrm{~cm}^{-1}(10 \%$ graphene in PSN-G), $6.09 \times 10^{-5} \mathrm{~S} \mathrm{~cm}^{-1}(10 \%$ graphene in AMDAFN-G), and $1.41 \times 10^{-4} \mathrm{~S} \mathrm{~cm}^{-1}$ (10\% graphene in POSSFN-G), respectively.

Tables S1-S3 list the work function data of the graphene-containing $3 \mathrm{D}$ composites with different graphene ratios on the ITO, evaporated gold and evaporated silver electrodes, measured with SKPM. The work function of ITO was set at $4.70 \mathrm{eV}$, and each value of the work functions was calculated relative to it. The evaporated gold and silver electrodes possess reasonable work functions of $4.65 \pm 0.03 \mathrm{eV}$ and $4.55 \pm 0.03 \mathrm{eV}$, respectively, which are the same as the reported values $[15,17,37]$. The work functions are reduced to $4.06-4.15 \mathrm{eV}$ for both ITO and the evaporated $\mathrm{Au}$ modified by PSN-G, and to
3.55-3.58 eV for the evaporated Ag modified by PSN-G. The work function of the AMDAFN-G with different graphene ratios is reduced about $0.60 \mathrm{eV}$ on ITO and $0.88-1.01 \mathrm{eV}$ on the evaporated $\mathrm{Ag}$ and $\mathrm{Au}$. Composites POSSFN-G shows reduced work functions of $0.42-0.64 \mathrm{eV}$ on the three different electrodes. All the work function values are lower than that of graphene $(4.60 \mathrm{eV})$ obviously [37], suggesting that the dispersed graphene into the CIMs still maintains the low work functions of the CIMs on the cathodes.

In order to further elucidate the charge transport properties of the graphene-containing composites CIMs, plane wave-based density functional theory calculations with periodic boundary conditions were performed $[24,25]$. The Bader charge values of different dispersants on single layer graphene are 0.001|e| for PSN, 0.04|e| for ADMAFN and 0.1|e| for POSSFN, respectively (as seen in Figs S1-S3). The degree of charge transfer trend is POSSFN-G $>$ ADMAFN-G $>$ PSN-G, which illustrates that the charge transport process exists between the $3 \mathrm{D}$ materials and graphene. To obtain more experimental evidence on the charge transport, the ESR spectra and the 
Raman spectra were measured. Fig. S13 presents the ESR spectra of the single components of PSN, ADMAFN, POSSFN and their composites with graphene. It can be seen that no obvious ESR signal was detected for POSSFN. With $10 \%$ graphene dispersed into the CIMs, the graphene-containing composites display broader and stronger resonance peaks with a $g$ value of 2.004 for both ADMAFN-G and POSSFN-G at room temperature under dark conditions. The ESR signal change of linear material PSN is significantly smaller than those of the $3 \mathrm{D}$ materials with dispersed graphene. Fig. 5a shows the Raman spectra of graphene flakes dispersed with different dispersants. The G-band shows a $1-4 \mathrm{~cm}^{-1}$ blue shift accompanied with a $3-7 \mathrm{~cm}^{-1}$ blue shift of the $2 \mathrm{D}$-band for the graphene samples dispersed with the dispersants [30-32,38]. In addition, the similar blue shifts were observed with the dispersants on CVD graphene on silica substrates (Fig. 5b shows the $0-3 \mathrm{~cm}^{-1}$ blue shifts for $\mathrm{G}$ band and $5-10 \mathrm{~cm}^{-1}$ blue shifts for 2D band) [38-41]. The Raman displacement of linear material PSN is significantly smaller than those of the 3D materials. Both the ESR and Raman results prove the existence of electron transfer, but whether the electron transfer exactly comes from the dipoles or ndoping effects remains to be further clarified.

XPS technique was performed to probe the composition and valence band changes induced by charge transfer (Table S4). For PSN, the N 1s binding energy appears at $399.5 \mathrm{eV}$ (Fig. 6a). As the graphene ratio in PSN increases, the $\mathrm{N}$ 1s binding energy of PSN in the composite moves from $399.5 \mathrm{eV}$ to higher value at $399.8 \mathrm{eV}$. Similar binding energy movement occurs in the $3 \mathrm{D}$ material ADMAFN, from $399.3 \mathrm{eV}$ for the pure ADMAFN to $399.8 \mathrm{eV}$ for the graphene-containing ADMAFN composite (raised by $0.5 \mathrm{eV}$ ), as seen in Fig. 6b. For the 3D material POSSFN, the $\mathrm{N}$ 1s binding energy of the amine group appears at $399.6 \mathrm{eV}$. As the graphene ratio in POSSFN increases, a new peak of the $\mathrm{N}$ 1s binding energy appears at $402.1 \mathrm{eV}$ and the ratio of $\mathrm{N} 1 \mathrm{~s}$ intensities at the peaks of $402 / 400 \mathrm{eV}$ increases with the increase of graphene content in the composites (Fig. 6c). This binding energy shift of the $\mathrm{N}$ 1s peak is quite different from the cases for ADMAFN and PSN where there are only 0.3-0.5 eV shifts. In POSSFN-G, there could be n-doping of POSSFN into graphene (charge transfer from POSSFN to graphene), while there is intermolecular dipole interaction involving electron donating from amine to graphene in the composites of ADMAFN-G and PSN-G. For the two 3D materials POSSFN and ADMAFN with the same functional amino groups, the different charge transfer mechanisms in their graphene-containing composites come from the pendant chain self-assembly of POSSFN which could donate more electrons from amine to graphene. Modeling results confirm more charge transfer in POSSFN-G.

In order to explain the charge extraction properties of the CIMs in the OSCs, the transient photocurrent (Fig. 7a) measurements were conducted for the PM6:Y6based devices. It is noteworthy that the charge extraction time for the devices with the graphene-containing composite CIM (0.47 $\mu$ s for PSN-G, $0.43 \mu$ s for ADMAFN-G and $0.46 \mu$ s for POSSFN) with a bias of $0 \mathrm{~V}$ (short-circuit condition) under $42 \mathrm{~mW} \mathrm{~cm}^{-2}$ illumination is much shorter than those of the devices with common CIMs $(0.66 \mu$ s for PSN, $0.63 \mu$ s for ADMAFN and $0.66 \mu$ s for POSSFN) and the device without the CIM $(0.78 \mu \mathrm{s})$. The faster charge carrier extraction in the composite CIMbased devices indicates that both $\mathrm{n}$-doping and diploe effect in the CIMs could decrease the charge extract time
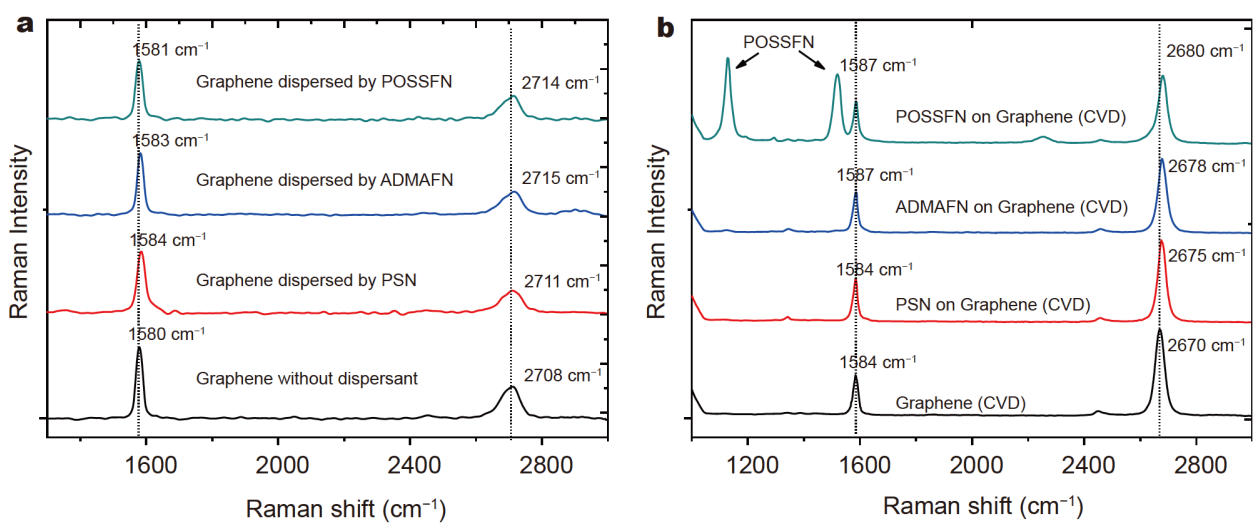

Figure 5 Full Raman spectrum for (a) graphene flakes dispersed with different dispersants, and (b) CVD graphene films treated without and with different dispersants of PSN, ADMAFN and POSSFN. 

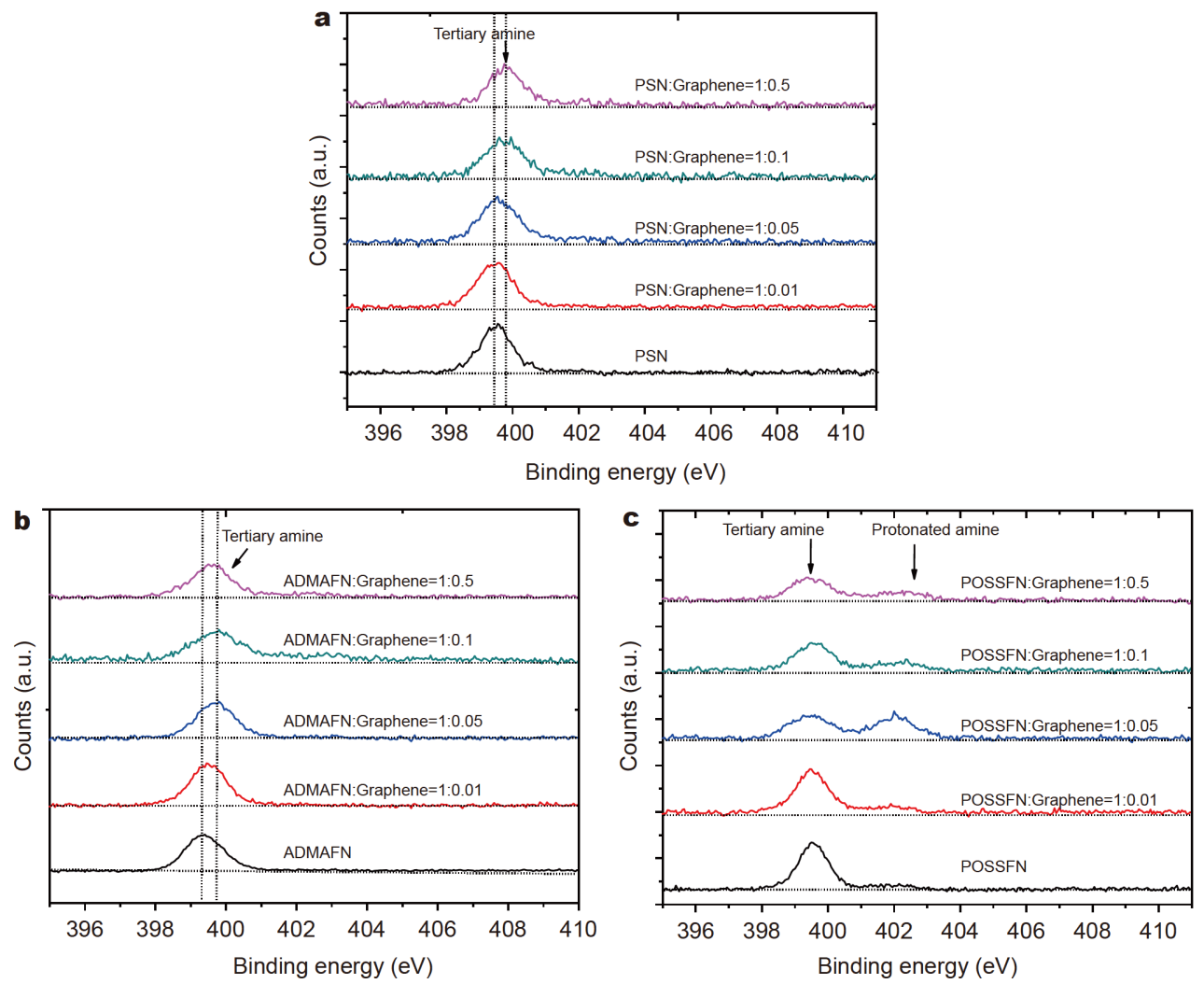

Figure 6 XPS spectra of the different cathode interfacial materials: N 1s XPS spectra for (a) PSN and PSN-G, (b) ADMAFN and ADMAFN-G, (c) POSSFN and POSSFN-G with different graphene ratios.
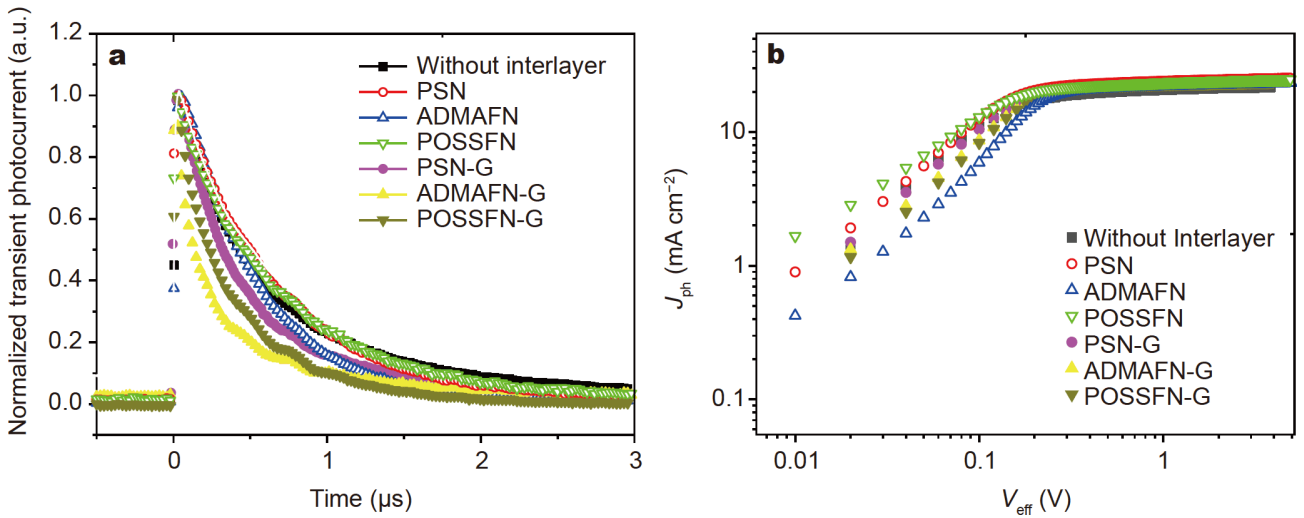

Figure 7 (a) Normalized transient photocurrent plots of the devices based on PM6:Y6 with different CIMs, under $42 \mathrm{~mW} \mathrm{~cm}^{-2}$ light bias. (b) Plots of photocurrent density $\left(J_{\text {ph }}\right)$ vs. effective bias $\left(V_{\text {eff }}\right)$ for the devices based on PM6:Y6 with different CIMs.

and mitigate the charge recombination.

Fig. $7 \mathrm{~b}$ shows the photocurrent density $\left(J_{\mathrm{ph}}=J_{\mathrm{L}}-J_{\mathrm{D}}\right.$, where $J_{\mathrm{L}}$ and $J_{\mathrm{D}}$ are the photocurrent densities under illumination and in the dark condition) versus effective bias $\left(V_{\text {eff }}=V_{0}-V_{\text {bias }}\right.$, where $V_{0}$ is the voltage at which $J_{\mathrm{ph}}=0$ and $V_{\text {bias }}$ is the applied bias) plot of the OSCs
$[42,43]$. The charge dissociation probability $(P(E, T))$ can be calculated from the value of the photocurrent density $J_{\mathrm{ph}}$ divided by the saturated photocurrent density $\left(J_{\text {sat }}\right.$, which is the saturated $J_{\text {ph }}$ value when $V_{\text {eff }} \geq 2 \mathrm{~V}$ ). Under the short circuit condition, the calculated $P(E, T)$ values are $96.17 \%, 97.04 \%, 97.49 \%$, and $96.35 \%$, for the OSCs 
with bare Al cathode, PSN CIM, ADMAFN CIM and POSSFN CIM, respectively. For the devices with different graphene-containing composite $\mathrm{CIMs}$, the calculated $P(E, T)$ values are $97.84 \%$ (PSN-G), 97.90\% (ADMAFN$\mathrm{G}$ ), and $97.89 \%$ (POSSFN-G), respectively. Since the $P(E, T)$ value is essentially the product of exciton dissociation efficiency and charge collection efficiency, the results indicate that the OSCs based on the 3D graphenecontaining composite CIMs have higher exciton dissociation rates and more efficient charge collection. The higher $P(E, T)$ values and the faster charge extraction could be responsible for the improved $J_{\mathrm{sc}}$ and $\mathrm{FF}$ of the OSCs with the graphene-containing CIMs.

\section{n-doping versus dipole interaction}

In order to check if there is charge transfer (n-doping) from the graphene-containing composite CIMs to the acceptors in the OSCs, the ESR spectroscopy was conducted under dark condition for the pure and the composite CIMs. No ESR signal was detected for the acceptor Y6 and the CIMs of PSN, ADMAFN and POSSFN. Then the blend samples of Y6 and the CIMs were prepared by dissolving them at a 1:1 weight ratio in chloroformethanol mixed solvent (0.1:1 volume) followed by drying under vacuum. As shown in Fig. S14a, strong ESR peaks with $g$ values of 2.005 were observed for the blend samples of PSN/Y6, ADMAFN/Y6 and POSSFN/Y6 at room temperature under dark condition. The ESR spectral intensity of the POSSFN/Y6 blend sample is significantly stronger than those of the ADMAFN/Y6 and PSN/Y6, probably because the different spatial structure and functional group density of the cathode interfacial materials could affect the charge transfer of lone-pair electrons [14-17]. Meanwhile, same ESR spectra with same $g$ values of 2.005 and intensity trend are displayed for the blend samples of PSN-G/Y6, ADMAFN-G/Y6 and POSSFN-G/Y6 (with 10 wt.\% graphene) under dark condition (as seen in Fig. S14b). The results indicate that the graphene-containing composites also have strong intermolecular $\mathrm{n}$-doping effect to Y6 acceptor.

The above results show that the ADMAFN-G works slightly better than the POSSFN-G on the device performance, even though the POSSFN-G possesses n-doped graphene and has higher conductivity than ADMAFN-G with only dipole interaction. As we have reported previously $[14,16]$, intermolecular $\mathrm{n}$-doping could occur between CIMs and acceptors in the active layer, and thus thin conductive interface could benefit the device performance. The intermolecular $n$-doping of the acceptor Y6 with ADMAFN-G could form a thin conductive in- terlayer near the active layer and contribute to the good device performance and the increased electron mobility. However, the n-doping of the acceptor could only take place near the interface. Thick ADMAFN-G film without n-doped graphene would have lower conductivity and may be unfavorable for the device performance. From Table S5, with the increase of the ADMAFN-G thickness from 3 to $12 \mathrm{~nm}$, the PCE of the device decreases gradually from $15.91 \%$ to $13.97 \%$. On the other hand, the POSSFN-G possesses $\mathrm{n}$-doped graphene and has higher conductivity. Its PCE keeps stable at about $15.65 \%$ when the thickness changes from 4 to $11 \mathrm{~nm}$. The tolerance to the CIM thickness is very important for future low-cost and large-area OSC fabrication.

\section{CONCLUSIONS}

Two 3D water/alcohol soluble CIMs of POSSFN and ADMAFN with amino functional groups were used for dispersing graphene with high concentration of $0.97-1.18 \mathrm{mg} \mathrm{mL}^{-1}$ in ethanol, benefitted from their large adsorption energies on graphene. The self-assembly performance of the pendant chain derives from the difference of spatial configurations of the materials. The amine fragment on POSSFN could n-dope graphene via selfassembly behavior, while the pendant chain segments spatially distributed on ADMAFN form dipole interaction with graphene. ADMAFN-G with dipole interaction delivers the highest PCE of $16.11 \%$, probably benefitted from the intermolecular $\mathrm{n}$-doping of the acceptor. As thicker CIM film in the OSCs, the n-doped POSSFN-G works better, attractive for future commercialization.

Received 13 April 2020; accepted 19 May 2020;

published online 21 July 2020

1 Yu G, Gao J, Hummelen JC, et al. Polymer photovoltaic cells: Enhanced efficiencies via a network of internal donor-acceptor heterojunctions. Science, 1995, 270: 1789-1791

2 Service RF. Outlook brightens for plastic solar cells. Science, 2011, 332: 293

3 Hou J, Inganäs $\mathrm{O}$, Friend RH, et al. Organic solar cells based on non-fullerene acceptors. Nat Mater, 2018, 17: 119-128

4 Lin Y, Wang J, Zhang ZG, et al. An electron acceptor challenging fullerenes for efficient polymer solar cells. Adv Mater, 2015, 27: $1170-1174$

5 Sun C, Pan F, Bin $\mathrm{H}$, et al. A low cost and high performance polymer donor material for polymer solar cells. Nat Commun, 2018, 9: 743

6 Fan Q, Su W, Wang Y, et al. Synergistic effect of fluorination on both donor and acceptor materials for high performance nonfullerene polymer solar cells with $13.5 \%$ efficiency. Sci China Chem, 2018, 61: 531-537

7 Zhao W, Li S, Yao H, et al. Molecular optimization enables over 13\% efficiency in organic solar cells. J Am Chem Soc, 2017, 139: 
7148-7151

8 Yuan J, Zhang Y, Zhou L, et al. Single-junction organic solar cell with over 15\% efficiency using fused-ring acceptor with electrondeficient core. Joule, 2019, 3: 1140-1151

9 Cui Y, Yao H, Zhang J, et al. Over 16\% efficiency organic photovoltaic cells enabled by a chlorinated acceptor with increased open-circuit voltages. Nat Commun, 2019, 10: 2515

10 Cui C, Li Y, Li Y. Fullerene derivatives for the applications as acceptor and cathode buffer layer materials for organic and perovskite solar cells. Adv Energy Mater, 2017, 7: 1601251

11 Wang C, Yang J. Interface modification for organic and perovskite solar cells. Sci China Mater, 2016, 59: 743-756

12 Wang W, He Y, Qi L. High-efficiency colorful perovskite solar cells using $\mathrm{TiO}_{2}$ nanobowl arrays as a structured electron transport layer. Sci China Mater, 2020, 63: 35-46

13 Lv M, Li S, Jasieniak JJ, et al. A hyperbranched conjugated polymer as the cathode interlayer for high-performance polymer solar cells. Adv Mater, 2013, 25: 6889-6894

14 Lv M, Li Y, Wei X, et al. Intermolecular n-doping nonconjugated polymer cathode interfacial materials for organic solar cells. ACS Appl Energy Mater, 2019, 2: 2238-2245

$15 \mathrm{Wu}$ Z, Sun C, Dong S, et al. n-Type water/alcohol-soluble naphthalene diimide-based conjugated polymers for high-performance polymer solar cells. J Am Chem Soc, 2016, 138: 2004-2013

16 Kang Q, Ye L, Xu B, et al. A printable organic cathode interlayer enables over $13 \%$ efficiency for $1-\mathrm{cm}^{2}$ organic solar cells. Joule, 2019, 3: 227-239

17 Novoselov KS, Geim AK, Morozov SV, et al. Electric field effect in atomically thin carbon films. Science, 2004, 306: 666-669

18 Geim AK, Novoselov KS. The rise of graphene. Nat Mater, 2007, 6: 183-191

19 Jeon IY, Shin YR, Sohn GJ, et al. Edge-carboxylated graphene nanosheets via ball milling. Proc Natl Acad Sci USA, 2012, 109: 5588-5593

20 Allen MJ, Tung VC, Kaner RB. Honeycomb carbon: A review of graphene. Chem Rev, 2010, 110: 132-145

21 Pan F, Sun C, Li Y, et al. Solution-processable n-doped graphenecontaining cathode interfacial materials for high-performance organic solar cells. Energy Environ Sci, 2019, 12: 3400-3411

22 Chen Y, Jiang Z, Gao M, et al. Efficiency enhancement for bulk heterojunction photovoltaic cells via incorporation of alcohol soluble conjugated polymer interlayer. Appl Phys Lett, 2012, 100: 203304

23 Lv M, Jasieniak JJ, Zhu J, et al. A hybrid organic-inorganic threedimensional cathode interfacial material for organic solar cells. RSC Adv, 2017, 7: 28513-28519

24 Junquera J, Paz Ó, Sánchez-Portal D, et al. Numerical atomic orbitals for linear-scaling calculations. Phys Rev B, 2001, 64: 235111

25 Troullier N, Martins JL. Efficient pseudopotentials for plane-wave calculations. Phys Rev B, 1991, 43: 1993-2006

26 Zhang L, Zhang Z, He C, et al. Rationally designed surfactants for few-layered graphene exfoliation: Ionic groups attached to electron-deficient $\pi$-conjugated unit through alkyl spacers. ACS Nano, 2014, 8: 6663-6670

27 Hernandez Y, Nicolosi V, Lotya M, et al. High-yield production of graphene by liquid-phase exfoliation of graphite. Nat Nanotech, 2008, 3: 563-568

28 Perumal S, Park KT, Lee HM, et al. PVP- $b$-PEO block copolymers for stable aqueous and ethanolic graphene dispersions. J Colloid Interface Sci, 2015, 464: 25-35
29 Zhang X, Coleman AC, Katsonis N, et al. Dispersion of graphene in ethanol using a simple solvent exchange method. Chem Commun, 2010, 46: 7539-7541

30 Chen W, Yan L. In situ self-assembly of mild chemical reduction graphene for three-dimensional architectures. Nanoscale, 2011, 3: 3132-3137

31 Skaltsas $\mathrm{T}, \mathrm{Ke} \mathrm{X}$, Bittencourt $\mathrm{C}$, et al. Ultrasonication induces oxygenated species and defects onto exfoliated graphene. J Phys Chem C, 2013, 117: 23272-23278

32 Ramanathan T, Abdala AA, Stankovich S, et al. Functionalized graphene sheets for polymer nanocomposites. Nat Nanotech, 2008, 3: 327-331

33 Chen J, Pan J, Huang Q, et al. Graphene oxide/PEDOT:PSS as injection layer for quantum dot light emitting diode. Phys Status Solidi A, 2015, 212: 2856-2861

34 Niu J, Yang D, Ren X, et al. Graphene-oxide doped PEDOT:PSS as a superior hole transport material for high-efficiency perovskite solar cell. Org Electron, 2017, 48: 165-171

35 Tung VC, Kim J, Cote LJ, et al. Sticky interconnect for solutionprocessed tandem solar cells. J Am Chem Soc, 2011, 133: 92629265

36 Blom PWM, Vissenberg MCJM. Charge transport in poly( $p$-phenylene vinylene) light-emitting diodes. Mater Sci Eng-R-Rep, 2000, 27: 53-94

37 Zhou Y, Fuentes-Hernandez C, Shim J, et al. A universal method to produce low-work function electrodes for organic electronics. Science, 2012, 336: 327-332

38 Graf D, Molitor F, Ensslin K, et al. Spatially resolved Raman spectroscopy of single- and few-layer graphene. Nano Lett, 2007, 7: 238-242

39 Movva HCP, Ramón ME, Corbet CM, et al. Self-aligned graphene field-effect transistors with polyethyleneimine doped source/drain access regions. Appl Phys Lett, 2012, 101: 183113

40 Bult JB, Crisp R, Perkins CL, et al. Role of dopants in long-range charge carrier transport for p-type and n-type graphene transparent conducting thin films. ACS Nano, 2013, 7: 7251-7261

41 Wei P, Liu N, Lee HR, et al. Tuning the Dirac point in CVD-grown graphene through solution processed n-type doping with 2-(2methoxyphenyl)-1,3-dimethyl-2,3-dihydro- $1 H$-benzoimidazole. Nano Lett, 2013, 13: 1890-1897

$42 \mathrm{Wu}$ JL, Chen FC, Hsiao YS, et al. Surface plasmonic effects of metallic nanoparticles on the performance of polymer bulk heterojunction solar cells. ACS Nano, 2011, 5: 959-967

43 Mihailetchi VD, Wildeman J, Blom PWM. Space-charge limited photocurrent. Phys Rev Lett, 2005, 94: 126602

Acknowledgements This work was supported by the National Natural Science Foundation of China (51820105003, 51863002 and 51973042), and the Excellent Young Scientific and Technological Talents of Guizhou, China (QKHPTRC [2019]5652).

Author contributions Lv M, Chen X and Li Y designed the interfacial modification materials; Bai S and Li Y synthesized and characterized interfacial modification materials; Pan F and Lv M carried out the PSCs fabrication and characterization. Tang D carried out the simulation calculations of absorption energies. Lv M and Li Y supervised the project. Lv M, Chen X and Li Y wrote the paper. All authors contributed to the general discussion.

Conflict of interest The authors declare no conflict of interest. 
Supplementary information Experimental details and supporting data are available in the online version of the paper.

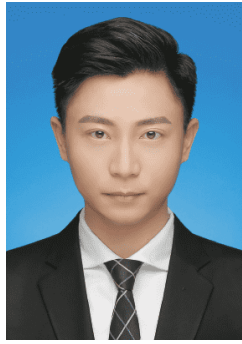

Fei Pan received his BSc degree in materials chemistry in 2016. Now he is a PhD candidate in Prof. Yongfang Li's group in the Institute of Chemistry, Chinese Academy of Sciences (ICCAS). His current research is the synthesis of cathode interfacial materials and their applications in organic solar cells.

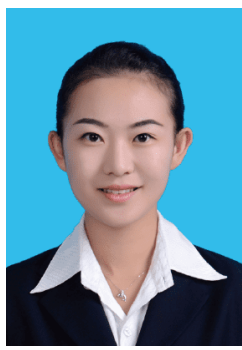

Menglan Lv received her $\mathrm{PhD}$ degree from Chengdu Institute of Organic Chemistry, Chinese Academy of Sciences in 2014. Currently, she is a professor of Guizhou Institute of Technology. Her research interest is the synthesis of cathode interfacial materials and their applications in organic solar cells.

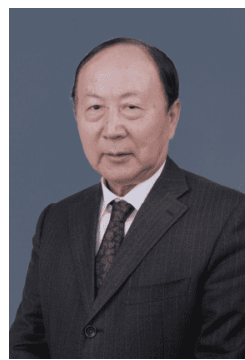

Yongfang $\mathbf{L i}$ is a professor in ICCAS and Soochow University. He received his $\mathrm{PhD}$ degree in Fudan University in 1986, and then did his postdoctoral research at ICCAS from 1986 to 1988. He became a staff in 1988 and promoted to professor in 1993 in ICCAS. He was elected as a member of the Chinese Academy of Sciences in 2013. His present research field is photovoltaic materials and devices for polymer solar cells.

\section{三维表面活性剂-石墨烯复合阴极界面材料在有 机太阳能电池中的应用 \\ 潘飞 ${ }^{1,2,4}$, 柏松 ${ }^{1}$, 魏娴 ${ }^{1}$, 黎应芬 ${ }^{1}$, 唐典勇 ${ }^{3}$, 陈希文 ${ }^{5}$, 吕梦岗 ${ }^{1,2^{*}}$, 李永舫 ${ }^{2,4^{*}}$}

摘要＼cjkstart低沸点绿色溶剂中分散石墨烯可广泛应用于涂料、导电油 墨、电池、电子产品和太阳能电池等领域. 研究发现, 在乙醇溶液 中, 两种三维阴极界面材料POSSFN和ADMAFN分散石墨烯的浓度 可达到0.97-1.18 mg mL $\mathrm{m}^{-1}$, 通过计算得知这两种材料在石墨烯表面 具有较大的吸附能. 通过ESR、Raman、SKPM和XPS等测试手段 证实, POSSFN侧链上的氨基能够与石墨烯发生 $\mathrm{n}$-掺杂作用; 而 $\mathrm{AD}$ MAFN与石墨烯之间能够形成偶极相互作用. 两种三维界面修饰石墨烯复合材料(POSSFN-G和ADMAFN-G)均可作为阴极界面修 饰材料应用于有机太阳能电池中，并使基于PM6:Y6光活性层的有 机太阳能电池的能量转换效率(PCE) 提高到了 $15.9 \%-16.1 \%$, 其中 基于存在偶极相互作用的复合材料ADMAFN-G阴极修饰层的有机 太阳能电池的PCE达到 $16.11 \%$; 使用存在n-掺杂作用的POSSFN-G 阴极修饰层的器件, 在厚膜状态下依然可获得较高的能量转换效 率. 